\title{
ON A CLASS OF FUNCTIONS SCHLICHT IN THE UNIT CIRCLE
}

\section{A. SCHILD}

Introduction. Actual tests to determine whether a regular function is schlicht in $|z|<1$ lead usually to tedious calculations of considerable difficulty. In this paper a class of polynomials having $|z|=1$ as radius of schlichtness is investigated, for which a rather simple condition-both necessary and sufficient-in terms of the coefficients is given. With the aid of this condition it is also possible to obtain for this class of polynomials better results for certain quantities connected with the conformal mapping of schlicht functions, such as: bound of convexity (Rundungs Schranke), distance of the boundary in the $w$-plane from $w=0$, etc.

Let $S_{p}$ be the class of functions having $|z|=1$ as radius of schlichtness, and let $f_{p_{-}}(z)=z-\sum_{n=2}^{N} a_{n} z^{n}$, having all $a_{n}$ real and non-negative for $n=2,3,4, \cdots, N, N \geqq 2$. Then we have:

THEOREM 1. A necessary and sufficient condition for $f_{p}(z) \in S_{p}$ is ${ }^{1}$

$$
1-\sum_{n=2}^{N} n a_{n}=0
$$

PROOF. To show sufficiency, suppose there exist $z_{1}, z_{2},\left|z_{1}\right|,\left|z_{2}\right|$ $\leqq \rho<1$, such that $f_{p}\left(z_{1}\right)=f_{p}\left(z_{2}\right)$. Then we have:

$$
\begin{aligned}
0 & =f_{p}\left(z_{1}\right)-f_{p}\left(z_{2}\right)=z_{1}-z_{2}-\sum_{n=2}^{N} a_{n}\left(z_{1}^{n}-z_{2}^{n}\right) \\
& =\left(z_{1}-z_{2}\right)\left\{1-\sum_{n=2}^{N} a_{n}\left(z_{1}^{n-1}+z_{1}^{n-2} z_{2}+\cdots+z_{2}^{n-1}\right)\right\} .
\end{aligned}
$$

But

$$
\begin{aligned}
\mid 1-\sum_{n=2}^{N} a_{n}\left(z_{1}^{n-1}+z_{1}^{n-2} z_{2}+\cdots\right. & \left.+z_{2}^{n-1}\right) \mid \\
& \geqq 1-\sum_{n=2}^{N} n a_{n} \rho^{n-1}>1-\sum_{n=2}^{N} n a_{n}=0
\end{aligned}
$$

and therefore $f_{p}\left(z_{1}\right)=f_{p}\left(z_{2}\right)$ implies $z_{1}=z_{2}$. To show that the condition is also necessary, we have: A necessary condition for a function to be

Presented to Society, April 24, 1953; received by the editors June 29, 1953.

1 The sufficiency of this condition is a well known result. It is proved here for the sake of completeness. 
schlicht in $|z|<1$ is that $f^{\prime}(z) \neq 0$ for $|z|<1$. But

$$
\left|f_{p}^{\prime}(z)\right|=\left|1-\sum_{n=2}^{N} n a_{n} z^{n-1}\right| \geqq 1-\sum_{n=2}^{N} n a_{n}|z|^{n-1}>1-\sum_{n=2}^{N} n a_{n} .
$$

Now, suppose $1-\sum_{n=2}^{N} n a_{n}<0$, then there exists a real $z=z_{0}$ such that $1-\sum_{n=2}^{N} n a_{n} z_{0}^{n-1}=f_{p}^{\prime}\left(z_{0}\right)=0$ since $f_{p}^{\prime}(0)=1$ and $f_{p}^{\prime}(1)<0$. Therefore, we must have $1-\sum_{n=2}^{N} n a_{n}>0$. But if $1-\sum_{n=2}^{N} n a_{n}>0$ then we would have schlichtness for $|z|=R>1$. Hence the condition is necessary.

Corollary. For functions $f_{p}(z)$ of class $S_{p}$ we have $a_{k} \leqq 1 / k, k$ $=2,3, \cdots, N$. If $a_{m}=1 / m$, then $f_{p}(z)=z-(1 / m) z^{m}$.

THEOREM 2. If $w=f_{p}(z) \in S_{p}$, then the map of $|z| \leqq 1$ cannot be $a$ convex region.

Proof. A necessary and sufficient condition that $w=f(z)$ should $\operatorname{map}|z| \leqq 1$ into a convex region is $\operatorname{Re}\left\{z f^{\prime \prime}(z) / f^{\prime}(z)+1\right\} \geqq 0$ for all $|z| \leqq 1$. For $f_{p}(z) \in S_{p}$ this condition becomes

$$
\operatorname{Re}\left\{\frac{1-\sum_{n=2}^{N} n^{2} a_{n} z^{n-1}}{1-\sum_{n=2}^{N} n a_{n} z^{n-1}}\right\} \geqq 0 .
$$

Now, considering the numerator of this expression we notice that for $z=0$ its value is positive and for $z=1$ its value is negative, since $1-\sum_{n=2}^{N} n^{2} a_{n}<1-\sum_{n=2}^{N} n a_{n}=0$. Therefore, there exists a real $z=z_{0}$ such that the numerator is equal to zero and will become and stay negative in an interval between $z=z_{0}$ and $z=1$, while the denominator stays positive (by Theorem 1), and therefore

$$
\operatorname{Re}\left\{\frac{1-\sum_{n=2}^{N} n^{2} a_{n} z^{n-1}}{1-\sum_{n=2}^{N} n a_{n} z^{n-1}}\right\}<0 \quad \text { for } z_{0}<z \leqq 1,
$$

which proves the theorem. However, we have:

THEOREM 3. All $w=f_{p}(z) \in S_{p}$ map $|z| \leqq 1$ into a region star-shaped with respect to $w=0$.

PROOF. A necessary and sufficient condition for $w=f(z)$ to map $|z| \leqq 1$ into a star-shaped region with respect to $w=0$ is 
$\operatorname{Re}\left\{z f^{\prime}(z) / f(z)\right\} \geqq 0$ for all $|z| \leqq 1$. In our case

$$
\operatorname{Re}\left\{\frac{z f^{\prime}(z)}{f(z)}\right\}=\operatorname{Re}\left\{\frac{1-\sum_{n=2}^{N} n a_{n} z^{n-1}}{1-\sum_{n=2}^{N} a_{n} z^{n-1}}\right\}=\operatorname{Re}\left\{1-\sum_{n=1}^{\infty} b_{n} z^{n}\right\}
$$

where $b_{1}=a_{2}$ and $b_{n}=n a_{n+1}+\sum_{k=1}^{n-1} b_{k} a_{n-k+1}$ for $n=2,3,4, \ldots$ It follows therefore by induction that all $b_{n}$ are positive. Therefore:

$$
\begin{aligned}
\operatorname{Re}\left\{\frac{z f^{\prime}(z)}{f(z)}\right\} & =\operatorname{Re}\left\{1-\sum_{n=1}^{\infty} b_{n} z^{n}\right\} \geqq \operatorname{Re}\left\{1-\sum_{n=1}^{\infty} b_{n}|z|^{n}\right\} \\
& \geqq 1-\sum_{n=1}^{\infty} b_{n}=0 .
\end{aligned}
$$

(Note: The last equality follows from the fact that the numerator of $\operatorname{Re}\left\{z f^{\prime}(z) / f(z)\right\}=0$ for $z=1$.) But $z f^{\prime}(z) / f(z)$ is regular for $|z| \leqq 1$, and therefore $\operatorname{Re}\left\{z f^{\prime}(z) / f(z)\right\}$ is a harmonic function, which satisfies $\operatorname{Re}\left\{z f^{\prime}(z) / f(z)\right\} \geqq 0$ on $|z|=1$. But a harmonic function cannot take a minimum "inside" and therefore $\operatorname{Re}\left\{z f^{\prime}(z) / f(z)\right\} \geqq 0$ for all $|z| \leqq 1$. This completes the proof.

THEOREM 4. If $w=f_{p}(z) \in S_{p}$ and $d^{*}$ is a point in the w-plane such that $f_{p}(z) \neq d^{*},|z| \leqq 1$, then $d^{*} \geqq 1 / 2$, i.e. the circle $|w| \leqq 1 / 2$ is always covered by the map of the unit circle by $f_{p}(z)=S_{p}$.

Proof.

$$
\left|f\left(e^{i \theta}\right)\right|=\left|e^{i \theta}-\sum_{n=2}^{N} a_{n} e^{i n \theta}\right| \geqq 1-\sum_{n=2}^{N} a_{n} \geqq 1-\frac{1}{2} \sum_{n=2}^{N} n a_{n}=\frac{1}{2} .
$$

This inequality is sharp, since for $f_{p}(z)=z-z^{2} / 2 \in S_{p}$ we have $f(1)=1 / 2$. We also note that if $f_{p}(z) \in S_{p}$ is of degree $k$, then $d^{*} \leqq 1-1 / k$ since $a_{2}+a_{3}+\cdots+a_{k} \geqq(1 / k) \sum_{n=2}^{k} n a_{n}=1 / k$.

THEOREM 5. The bound of convexity of $w=f_{p}(z) \in S_{p}$ is $1 / 2$, i.e. $\left|\begin{array}{l}z \\ z\end{array}\right|=r>1 / 2$.

PROOF. The bound of convexity is the least zero-in absolute valueof $\operatorname{Re}\left\{z f^{\prime \prime}(z) / f^{\prime}(z)+1\right\}=\operatorname{Re}\left\{\left(1-\sum_{n=2}^{N} n^{2} a_{n} z^{n-1}\right) /\left(1-\sum_{n=2}^{\infty} n a_{n} z^{n-1}\right)\right\}$ $=1-\sum_{n-1}^{\infty} c_{n} z^{n}$ where all $c_{n} \geqq 0$. By considerations similar to those of Theorem 3 , we find that the least zero will be a positive real num- 
ber $0<z_{0}<1$. We must have, therefore, a $z_{0}$ such that 1 $-\sum_{n=2}^{N} n^{2} a_{n} z_{0}^{n-1}=0$.

Suppose now $z_{0}<1 / 2$; then $1-\sum_{n=2}^{N} n^{2} a_{n}(1 / 2)^{n-1}<0$. But

$$
\begin{aligned}
1-4 a_{2} \cdot \frac{1}{2}-9 a_{3} \cdot \frac{1}{4}-\cdots-N^{2} a_{N} \frac{1}{2^{N-1}} \\
=1-2 a_{2}-\frac{9}{4} a_{3}-\frac{N^{2} a_{N}}{2^{N-1}}>1-2 a_{2}-3 a_{3}-\cdots-N a_{N}=0
\end{aligned}
$$

which is a contradiction. The estimate is exact since for $f_{p}(z)=z$ $-z^{2} / 2$ we have $r_{0}=1 / 2$.

THEOREM 6. The circle $|w| \leqq 3 / 8$ is always covered by a convex region, i.e. if $d_{0}$ is the shortest distance from $w=0$ to $f_{p}\left(r_{0} i^{i \theta}\right)$, where $r_{0}$ is the bound of convexity, then $d_{0} \geqq 3 / 8$.

PROOF. $\quad d_{0}=\left|f\left(r_{0} e^{i \theta}\right)\right|=\left|r_{0} e^{i \theta}-\sum_{n=2}^{N} a_{n} r_{0}^{n} e^{i n \theta}\right| \geqq r_{0}-\sum_{n=2}^{N} a_{n} r_{0}^{n}$ $=r_{0}\left\{1-\sum_{n=2}^{N} a_{n} r_{0}^{n-1}\right\} \geqq r_{0}\left\{1-r_{0} \sum_{n=2}^{N} a_{n}\right\} \geqq r_{0}\left\{1-r_{0} / 2\right\}$. We know by the previous theorem that $r_{0} \geqq 1 / 2$. But $r_{0}\left\{1-r_{0} / 2\right\}$ is strictly increasing in $1 / 2 \leqq r_{0}<1$, therefore

$$
d_{0} \geqq \frac{1}{2}\left\{1-\frac{1 / 2}{2}\right\}=\frac{3}{8} \text { q.e.d. }
$$

For the function $f(z)=z-z^{2} / 2$ this estimate is exact. By Theorems 4 and 6 we have

$$
1 / 2 \leqq d^{*}<1, \quad 3 / 8 \leqq d_{0}<d^{*}
$$

and therefore obviously

$$
d_{0} / d^{*}>3 / 8
$$

It has been conjectured that for all functions $w=f(z)=z+\sum_{n=2}^{\infty} a_{n} z^{n}$ regular and schlicht in the unit circle which map $|z| \leqq 1$ into a starshaped region we have $d_{0} / d^{*} \geqq 2 / 3$. (This lower bound for $d_{0} / d^{*}$ cannot be improved since, for $f(z)=z(1+z)^{-2}, d^{*}=1 / 4, d_{0}=1 / 6$.) It is possible to prove this conjecture for all $f(z) \in S_{p}$.

THEOREM 7. For all $f_{p}(z) \in S_{p}$ we have $d_{0} / d^{*} \geqq 2 / 3$.

PROOF.

$$
d_{0}=\int_{0}^{z_{0}} f_{p}^{\prime}(z) d z \geqq z_{0} f_{p}^{\prime}\left(z_{0}\right)
$$


since $f_{p}^{\prime}(z)$ is decreasing for $0 \leqq z \leqq z_{0}, \operatorname{Im} z=0$. Also

$$
d^{*}=\int_{0}^{1} f_{p}^{\prime}(z) d z=\int_{0}^{z_{0}} f_{p}^{\prime}(z) d z+\int_{z_{0}}^{1} f_{p}^{\prime}(z) d z=d_{0}+\int_{z_{0}}^{1} f_{p}^{\prime}(z) d z .
$$

But for all $f_{p}(z) \in S_{p}, f_{p}^{\prime \prime \prime}(z) \leqq 0$ for $0 \leqq z \leqq 1, \operatorname{Im} z=0$, i.e. $f_{p}^{\prime}(z)$ is convex upward in this interval and therefore the tangent to $f_{p}^{\prime}(z)$ at $\left(z_{0}, f^{\prime}\left(z_{0}\right)\right)$ will lie entirely above the $f_{p}^{\prime}(z)$ curve. But at $\left(z_{0}, f^{\prime}\left(z_{0}\right)\right)$ we have $z_{0} f^{\prime \prime}\left(z_{0}\right) / f^{\prime}\left(z_{0}\right)+1=0$, i.e. $f^{\prime \prime}\left(z_{0}\right)=-f^{\prime}\left(z_{0}\right) / z_{0}$, i.e. the slope of the tangent at $\left(z_{0}, f^{\prime}\left(z_{0}\right)\right)$ is $=-f^{\prime}\left(z_{0}\right) / z_{0}$, and therefore the tangent will intersect the $\operatorname{Re} z$-axis at $z=2 z_{0}$. Therefore $\int_{z_{0}}^{1} f_{p}^{\prime}(z) d z \leqq$ area of the triangle formed by the three points:

$$
\left(z_{0}, f^{\prime}\left(z_{0}\right)\right), \quad\left(z_{0}, 0\right), \quad\left(2 z_{0}, 0\right),
$$

i.e. $\int_{z_{0}}^{1} f_{p}^{\prime}(z) d_{z} \leqq z_{0} f_{p}^{\prime}\left(z_{0}\right) / 2$. Thus $d^{*} \leqq d_{0}+z_{0} f_{p}^{\prime}\left(z_{0}\right) / 2$ and on account of (1)

$$
d^{*} \leqq d_{0}+d_{0} / 2=3 d_{0} / 2 \text {, i.e. } \quad d_{0} / d^{*} \geqq 2 / 3 .
$$

It seems highly probable that, for $f_{p}(z) \in S_{p}$, we actually have $d_{0} / d^{*} \geqq 3 / 4$, which could not be improved, since it is sharp for the function $f_{p}(z)=z-z^{2} / 2$.

THEOREM 8. For the area of the map of $|z| \leqq 1$ by $f_{p}(z) \in S_{p}$, we have

$$
\pi<A \leqq 3 \pi / 2 \text {. }
$$

Proof. The left inequality is obvious, since $A=\pi\left\{1+\sum_{n=2}^{N} n a_{n}^{2}\right\}$. To prove the right-hand side of the inequality, we have:

$$
\begin{aligned}
\pi\left\{1+\sum_{n=2}^{N} n a_{n}^{2}\right\} & \leqq \pi\left\{1+\sum_{n=2}^{N} n \cdot \frac{1}{n} \cdot a_{n}\right\}=\pi\left\{1+\sum_{n=2}^{N} a_{n}\right\} \\
& \leqq \pi\left\{1+\frac{1}{2}\right\}=\frac{3}{2} \pi .
\end{aligned}
$$

This is exact, since for $f_{p}(z)=z-z^{2} / 2, A=3 \pi / 2$.

THEOREM 9. For the functions $f_{p}(z)$ of class $S_{p}$ we have the following distortion theorem:

$$
|z|-|z|^{2} / 2 \leqq\left|f_{p}(z)\right| \leqq|z|+|z|^{2} / 2 \text {. }
$$

Proof. $|f(z)|=\left|z-\sum_{n=2}^{N} a_{n} z^{n}\right| \leqq|z|+\sum_{n=2}^{N} a_{n}|z|^{n} \leqq|z|+|z|^{2}$ - $\sum_{n=2}^{N} a_{n} \leqq|z|+|z|^{2} / 2$.

Similarly, $\quad\left|f_{p}(z)\right| \geqq|z|-\sum_{n-2}^{N} a_{n}|z|^{n} \geqq|z|-|z|^{2} \sum_{n-2}^{N} \quad a_{n} \geqq|z|$ $-|z|^{2} / 2$. 
ThEOREM 10. For the derivatives of functions $f_{p}(z)$ of class $S$ :

$$
1-|z| \leqq\left|f_{p}^{\prime}(z)\right| \leqq 1+|z| \text {. }
$$

These inequalities are sharp. They are attained by $f_{p}(z)=z-z^{2} / 2$ at $z= \pm r, r$ real.

TEMPLE UNIVERSITY

\section{A REMARK ON REVERSIBLE MATRICES}

\section{S. MACPHAIL}

The matrix $A=\left(a_{n k}\right)$ is called reversible if for each $y=\left\{y_{n}\right\} \in(c)$ the equations $y_{n}=\sum_{k=0}^{\infty} a_{n k} x_{k}(n=0,1, \cdots)$ have exactly one solution $x=\left\{x_{k}\right\}$. In this case there exist $[1 ; 4]$ constants $c_{k}, b_{k n}$ with $\sum_{n}\left|b_{k n}\right|<\infty$ for each $k$, such that

$$
x_{k}=c_{k} \lim _{n \rightarrow \infty} y_{n}+\sum_{n=0}^{\infty} b_{k n} y_{n} \quad(k=0,1, \cdots) .
$$

It is further stated in $[1$, p. 50$]$ that the $c_{k}$ are bounded. This is questioned in [4], where it is pointed out that if the $c_{k}$ were generally bounded they would have to be almost all zero, but this remark does not dispose of the matter, for it might conceivably be a true theorem that for each reversible matrix the $c_{k}$ are almost all zero. (For rowfinite matrices, all $c_{k}$ vanish; [3].) The example given in [4, p. 47] seems inconclusive. The purpose of this note is to show by a very simple example that in fact the $c_{k}$ need not be bounded.

Consider the transformation

$$
y_{2 m}=\sum_{p=0}^{m} x_{2 p}, \quad y_{2 m+1}=2^{-m} x_{2 m+1}+\sum_{p=0}^{\infty} x_{2 p},
$$

where $m=0,1, \cdots$. For each $y \in(c)$ we have

$$
x_{2 m+1}=2^{m}\left(y_{2 m+1}-\lim _{n} y_{n}\right)
$$

thus $c_{2 m+1}=-2^{m}$ is not bounded.

This has a bearing on a paper [2] in which the following theorem is stated:

Received by the editors June 22, 1953. 